\title{
An Overview of the Development of Chinese Embroidery Patterns in the Past Dynasties
}

\author{
Xianrong $\mathrm{Yu}$ \\ Ganzhou teachers college Ganzhou Jiangxi China.
}

\begin{abstract}
Embroidery patterns is a special language to express Chinese traditional culture and human aesthetic concept, and also an important carrier for inheriting Chinese traditional culture and humanistic connotation. It is not only the crystallization of human wisdom for many years, but also a bright pearl in the treasure house of human material culture. China's Embroidery decorative patterns have a long history and long history. All kinds of decorative patterns have already existed in the costumes of the Xia Dynasty. People integrate their life habits, religious beliefs, aesthetic sentiment into their own costumes, and build a colorful cultural connotation of national costumes. Through the analysis of the evolution of the style of the embroidery decorative patterns, this article further excavates the cultural connotation of the traditional costumes of our country and explores the development law of the traditional embroidered decorative patterns in our country, so that people can know more about the cultural connotation of the clothing behind the profound and profound culture of our Chinese nation.
\end{abstract}

Key words: Embroidery patterns; historical development; tradition; style; change.

\section{中国历代织绣纹样发展概述}

\author{
余先荣 \\ 赣州师范高等专科学校, 江西赣州 中国
}

摘要: 织绣纹祥是表达中华传统文化与人类审美观念的特殊语言, 也是传承中华传统文化和 人文内涵的重要载体。它既是人类多年来智慧的结晶, 也是人类物质文化宝库中一颗璀骤的 明珠。我国织绣装饰纹样发展历史悠久, 源远流长, 早在夏朝的服饰中就已经存在各种各样 的装饰纹样, 人们将自己的生活习惯、宗教信仰、审美情操等融入到自己的服饰当中, 构建 出丰富多彩的民族服饰文化内涵。本文通过对织绣装饰纹样风格演变的分析, 进一步挖掘我 国传统服饰的文化底蕴, 探求我国传统织绣装饰纹样的发展规律, 使人们更加了解我们中华 民族博大精深文化背后的服饰文化内涵。

关键词: 织绣纹样; 历史发展；传统；风格；变迁

\section{1. 前言}

我国古代服饰的纹样拥有源远流长的历史, 且服饰的纹样都源自于人们的生活, 同时也蕴含 着当时人们的审美观念, 并且随着社会的发展而发展。起初, 中国古代服饰的装饰纹样多以 用简单的几何图案、动物、植物为主, 但随着时代的发展, 其纹样的表现形式由抽象派, 也 逐渐演变成为了写实派。例如在商、周代之前, 其织绣装饰纹样跟原始的汉字如出一辙, 表 现形式也极其简捷、干练, 同时具有较强的抽象感。从周朝以后, 一直到唐宋时期, 织绣装 饰纹样呈现工整、均衡、对称的布置模式, 且其纹样布局较为严密。等到了明清时期, 人们 的审美观念受到西方国家的影响, 大有转变, 在织绣装饰纹样的编排上, 创作者更加注重写 实的表现手法, 将各种动物、植物或是人物刻画得栩栩如生、细淢逼真。正所谓 “艺术来源 于生活” , 纵观我国历朝历代的织绣装饰纹样, 其图案都直接采撷于当代人民的现实生活, 也是我国文化发展的真实写照。 


\section{2. 商代时期的服饰纹样}

商代图案在服饰的表现上, 主要以纹路为主。如简单的、动物纹路（回龟纹、狮兽纹）以及 最为常见的云雷纹。云雷纹在服装上是以主纹的形式存在的, 一般采用两方连续的构图方式, 并且工匠也非常重视其造型的规律美。一开始的云雷纹是以单一的 S 形体存在的, 随着造匠 审美观念的不断改变, 单个的 S 型云雷纹逐渐发展成为一个单位的纹样与另一个单位纹样的 连续组合的形式, 即多个单个子体的相互组合, 如图 1 所示。

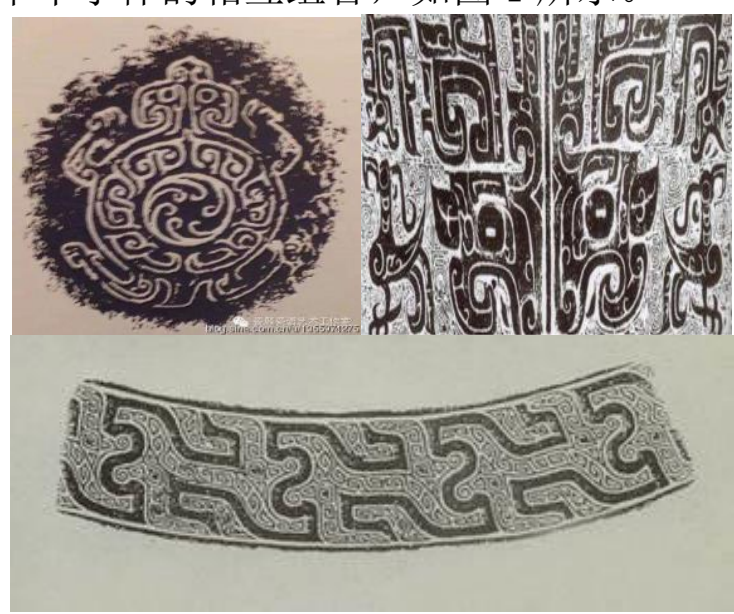

图1 商朝时期服饰中的纹样

在之后的纹样造型中, 工匠们又将其主观意识进行夸张式的处理, 例如, 将类似 $\mathrm{S}$ 型进行类 似倒放设置，这样的纹样主要在服饰的领口、袖口、下摆等边缘处进行布置，体现当时人们 对细致、高雅生活的追求。纵观奴隶社会到封建社会的服饰上的装饰纹样, 我们可以得知, 当时的匠人主要都是从对于动物、形状获取创作灵感, 虽然其主观色彩不够浓厚, 但其是真 实自然物象的一种体现，体现出当时我国古代劳动人民的淳朴品质。

\section{3. 周代时期的服饰纹样}

从商朝到周朝, 社会的生产力不断增强, 纺织业也得到了快速的发展, 于是出现了很多华丽 的刺绣品。人们利用这一华丽的刺绣纹饰来制作服装, 进而出现了 “冕服”。据史书记载的 十二章纹样, 它们分别是日、月、星、龙、山、华虫、火、宗彝、藻、粉米、糐、䊑, 并且 它们都存在各自的含义和象征。日、月、星三个纹样象征着光明; 人们称之为 “三光之耀” ; 龙, 具有浓郁神话气息, 取其神意, 代表着万物的变化; 山, 象征着镇压四方的祥和; 华虫, 表示帝王具有文章之德; 宗彝：代表威猛之德；火，一般取其明；粉米，具有洁白、养人 之意; 䊇, 含断割之意; 䟦, 其意为见恶改善, 也有具有背离邪恶的含意; 十二章纹样是寓 意纹，当时人们是观天地万物之景像，择之用于服饰之上。

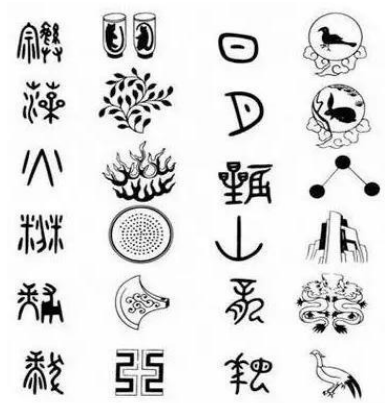

图 2 十二章纹样

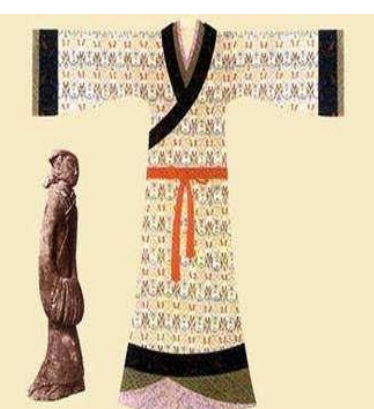

图3 周代时期服饰纹样

从十二章纹样表现的形式和象征意义来看，人们从天地万物间寻来的纹样都能够揭示出当时 人们对图腾的崇拜心理，同时，还表明人们不单单将图腾崇拜作为服饰纹样装饰，并且利用 各种自然界的纹样来装饰自己, 表达自己的心理诉求, 进而表现出了奴隶社会下人们的审美 意识。而这样的审美意识又普遍存在着一定的局限性, 在奴隶主与奴隶之间存在一定的差异 
性。换句话说, 统治阶级有意识的将纹样划分出等级, 使本来同属于大自然的景象纹样蒙上 了浓厚的阶级色彩。例如, 天子服饰可尽享十二章花纹; 而士服只可以藻、火, 大夫加粉米 为纹样。由此我们可以看到, 商朝时期的服饰纹样以及开始注重艺术的表达, 也表明了当时 人们审美意识正在提高。

\section{4. 春秋战国时期的服饰纹样}

战国时期, 由于百家争鸣, 战乱频发, 导致社会思潮空前活跃。此时器物的装饰纹样较前朝 巨有较大的变化, 例如森严拘谨的饕䬸纹、蟠螭纹, 这些纹样的表现形式开打了让当时工匠 们的创造思路, 在服饰上的设计变得舒展得多。从战国的服饰图案来看, 服饰上的纹样并不 是直接利用自然物象进行直观描绘, 反而将自然物象进行相应的变化, 演变成为具有创意彩 色的纹样，如图 4、5 所示。

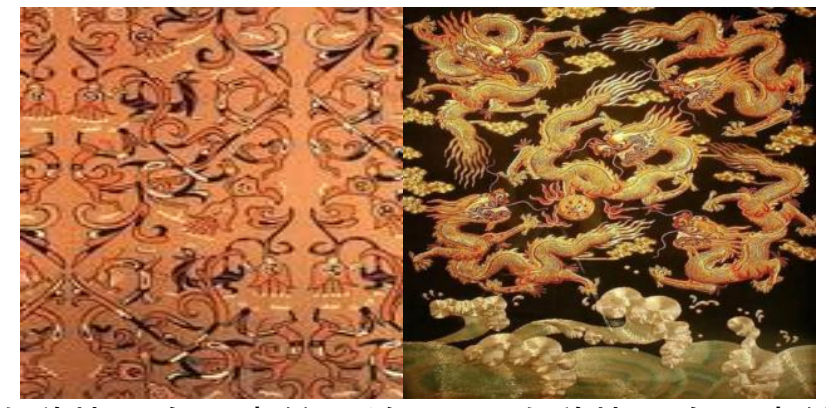

图4 春秋战国龙风虎纹经绵 图5 春秋战国龙风虎纹刺绣

如上图所示, 当时人们对于服饰纹样的设计, 都加入了自己主观的审美观念, 将纹样变得更 加的理想化。这些在潜移默化中都表现出了当时的人们审美情趣正在逐步提高, 对于现在的 艺术品鉴而言, 具有较高的艺术研究价值。云纹瓦是当时最为流行的服饰纹样之一, 给人以 大气、磅礴的感觉, 看似这些纹路没有差别, 但在布置的变化规律上又强调了一个 “变” 字, 细细品味之后又各持风格, 在春秋战国时期的服装纹样上得到了广泛应用。

\section{5. 秦汉时期的服饰纹样}

秦汉时期的服饰纹样，秉承了战国时期的服饰风格，在其纹样的设计上更加显得大气磅礴、 简练多变。不再运用商、周朝代的中心对称以及连续拼接式的布置形式, 多半是以重叠缠绕、 上下穿插、四面延展的构图进行呈现, 并增添了浪漫虚幻的艺术色彩, 在原有简单的几何图 文、动、植物图文上进行生动的变形, 形成了具有生机的雷云纹、鸟兽纹或祥龙纹等。这样 的纹样比起夏、商、周朝代的看着更加舒展大方。秦朝服饰纹样的色彩, 主要是以对比形式 为主, 以此来增加服饰的醒目艳丽感, 典型的 “素中见华美”, 其影响意义非常深远, 在现 代服饰设计中也会被人们所运用。而自从大秦王朝崩土瓦解之后, 汉朝经久不衰, 统治时长 四百余年, 汉武帝传承秦始皇的思想, 祈求盼望长生不老, 子孙延绵, 皇权不倒。这样的思 念很快在当时的服饰纹样中呈现出来, 多半结合神话寓言、仙鹤猛禽、日月星辰等进行服饰 纹样的创作, 从新疆楼兰遗迹出土的秦汉服饰纹样来看, 都充斥着宗教信仰和神话色彩。 汉代服饰图案除了对十二章纹样的继续延用之外, 在纹样造型的选择方面, 将龙、风、火的 寓意进行深化。在服饰纹样的表现形式上, 常常以物、植物纹样或是雷云纹进行工艺化、装 饰化, 具有更高的艺术审美价值, 如图 6 所示。 


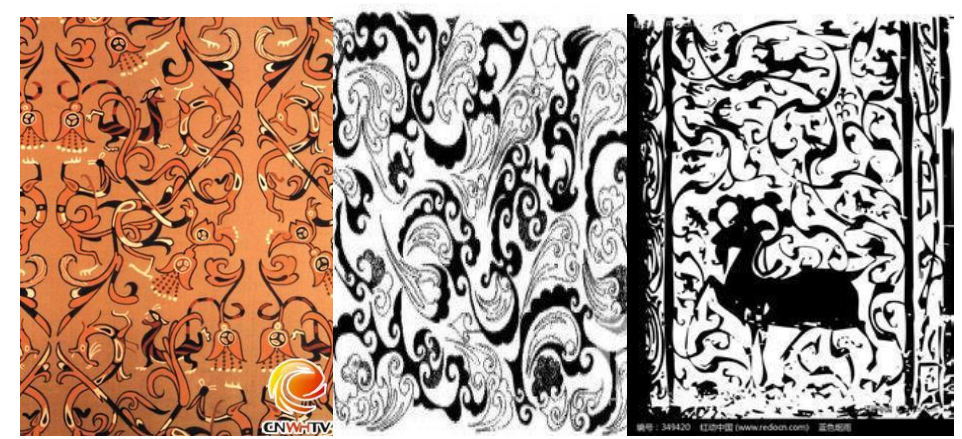

图6 秦汉服饰纹绣

\section{6. 魏晋、南北朝时期的服饰纹样}

汉代绵织纹样在形式上不再局限于动物的写实, 它将人类与动物进行主观意识上的结合, 即 捕捉到事物的细节进行刻画，再抓住形象的宏观结构，展现出活跃蹦腾、气魄恢宏的画面。 而在魏晋时期，由于君王没有对服饰提出新的要求，使得其所采用的装饰纹样没有较大的变 化, 仅仅在秦朝纹样的工艺上稍作更改, 使得汉代的云端动物样式的纹饰时过境迁, 逐渐衰 退, 魏晋时期的纹饰样式如图 7、8 所示。

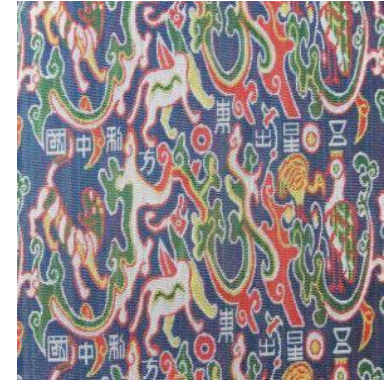

图7 北朝云气动物纹锦

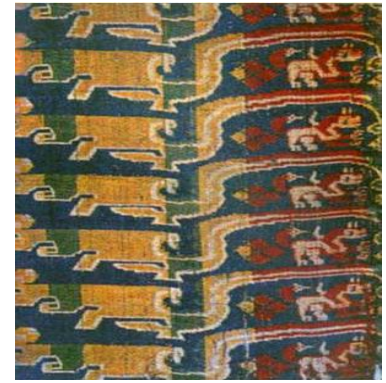

图8 魏晋忍冬几何纹

\section{7. 隋朝时期的服饰纹样}

到了隋代，由于隋炀帝登基，造成社会风气大变，其服饰纹样呈现出华贵之风，在服饰中运 用最多的还是云纹样。从图 9 可以看出, 在服饰装饰上, 隋代服饰图案具有惊人的艺术成就, 服饰纹样采用联珠纹服饰图纹、狮凤纹服饰图纹以及团花织锦图案纹样，色显金贵，迷人大 方。这雷图纹案式是隋代服饰图案的典型代表作品，且工匠艺人手法精妙，技艺高超，以对 称、连续、交错进行排列, 纹样意象清晰动人, 是我国织绣服饰纹样中不可多得的精品。

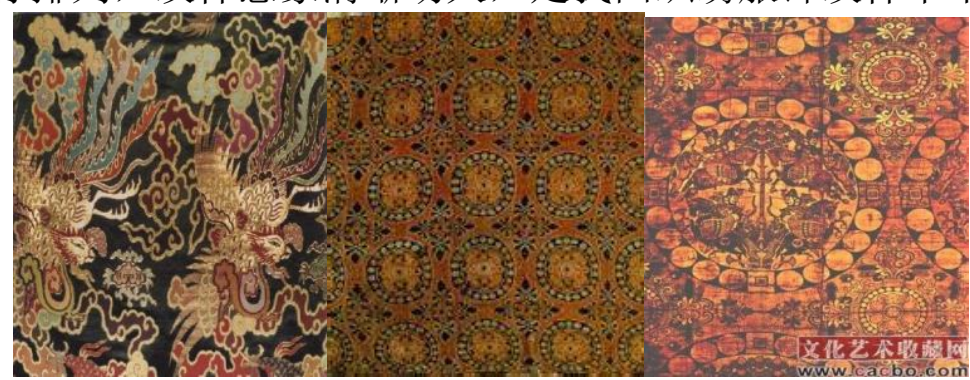

图9 隋朝服饰纹样

\section{8. 唐朝时期的服饰纹样}

唐代, 服饰纹样普遍利用花卉纹样进行装饰, 构图疏密匀称、活泼自由。尤其是花草相结合 后的波状连续纹样，即盛唐时期的缠枝图纹。在唐代服饰纹样创作上，改变了奴隶社会的那 
种 “天赋神授” 的创作思想，而是利用现实中花、草、鱼、虫进行写生，但传统的龙凤图案 并没有受到排斥, 皇权贵族仍旧以龙纹的纹样作为黄袍上的主要图案, 如图 10 所示。而到了 晚唐时期, 其服饰图案变得更加的精巧、美观，对后世的服饰设计产生了不小的影响，展现 出了传统纹样与现代审美意识结合所产生的意蕴之美。

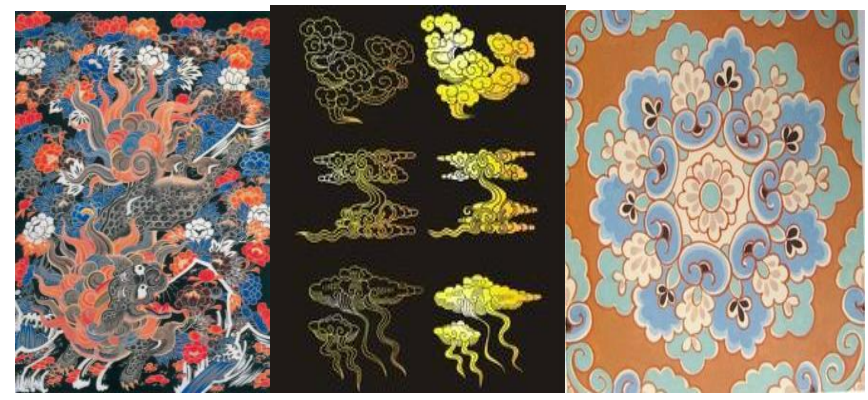

图10 唐代龙、云服饰纹样

\section{9. 宋、元、明、清时期的服饰纹样}

宋、元、明、清时期的服饰图案在纹样本质上都继承了唐朝时期的写实风格, 在不同的纹样 设计上展现出了不同的装饰风格。这时期的服饰, 在纹样的运用上不仅将简单的云纹变成了 朵云纹样, 而且还存在 “吉祥” 的寓意, 人们大量运用 “吉祥” 式的图纹, 将传统的云纹和 回龟纹进行变形，最终给人以简明、厉练的感觉，如图 11、12、13 所示。在 “吉祥图案” 的 影响下, 人们将云纹样逐渐发展成为一种 “如意云” 图案, 并将几个如意云拼接在一起, 增 加了纹样装饰的趣味; 还有一种变化的发生, 即将 “自由式” 的云纹样逐渐演变成为 “写生 式”的云纹样，明、清朝代中云锦图案的云纹，就是很典型的作品。

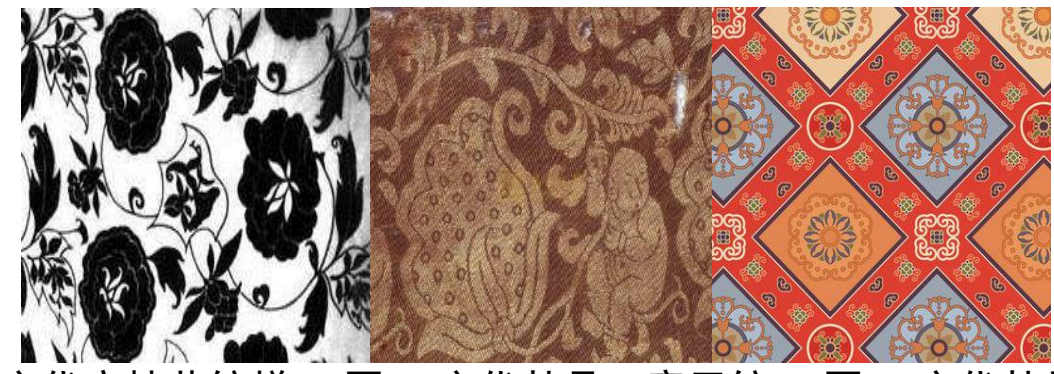

\section{图11 宋代穿枝花纹样 图12 宋代牡丹、童子纹 图13 宋代牡丹纹绫}

到了明朝，纹样的设计更加富有寓意。在继承前代纹样风格的同时，创造出了“谐音图纹” 和 “寓意图纹”。例如经典的 “万年青”，寓意富贵常在；而太阳和凤凰，则意为 “丹凤朝 阳”; 莲花和鲤鱼, 就被寓意为我们熟知的 “连年有余” 等等。这种具有吉祥气息的寓意图 案，有的至今沿用，如图 14、15 所示。

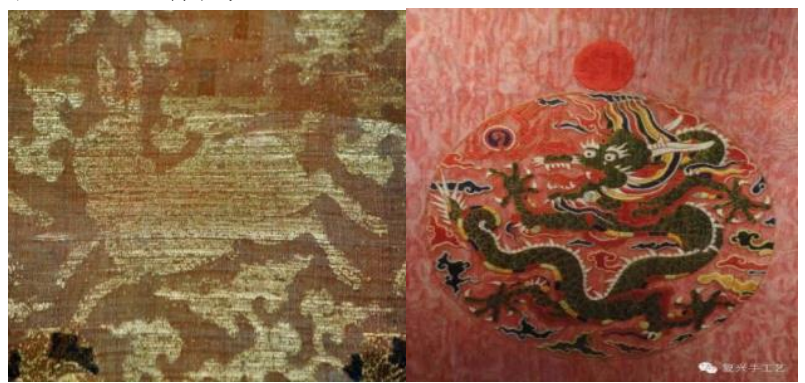

图14 明代妆花奔兔纱 图15 明孔雀羽织金妆花纹

清代服饰中的纹样繁华富丽, 例如清代皇后所穿的服饰, 如图 16 所示。其所采用的服装纹样 是 “丹凤穿牡”。整件服装在蓝色的䌸地上, 绣了八只形象不同的彩凤，彩凤中间穿插着数 朵牡丹。匠人在对牡丹颜色的处理上非常净穆, 色彩变化微秒, 与现代山水画有异曲同工之 妙。在对边景的处理上, 颜色显得较为浓厚凝重, 形成鲜明的对比度极, 具有鲜明的满族风 格和时代特色。 

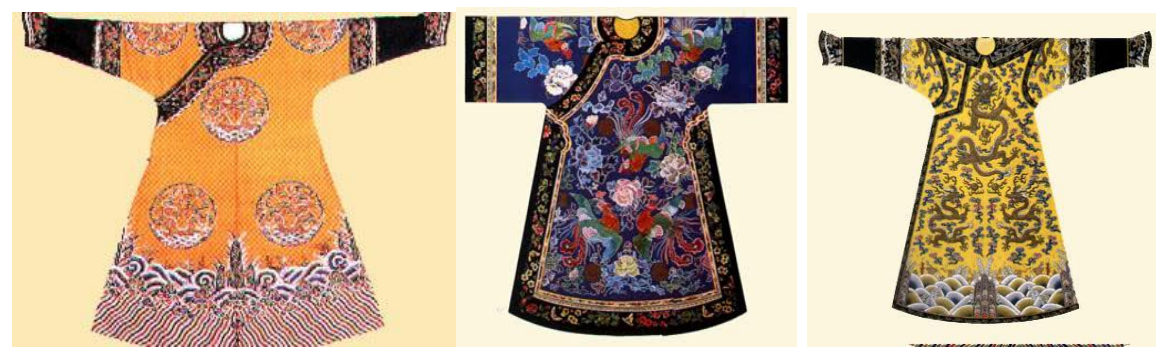

图16 清代皇后服饰纹样

\section{References}

[1]. Jin Kaicheng. China eaving embroidery [M]. Jilin literature and history press, 2012.

[2]. Liu Yuanyang. Ancient Chinese embroidery and embroidery patterns [M]. Xue Lin press, 2016.

[3]. Xing Lu. Study on the embodiment of aesthetics in ancient Chinese costume patterns [D]. Shenyang Jianzhu University, 2014.

[4]. Liu Yang. Analysis of the group pattern in Tang Dynasty figure painting costumes [D]. Harbin Normal University, 2013.

[5]. Li Yujuan. The decoration and application of Four Seasons flower pattern in women's dress in the late Qing Dynasty and its innovative application [D]. Beijing Institute of Fashion Technology, 2017.

[6]. Zhang Shuhua. Research on the fitting pattern of the festival costume in the Ming Dynasty and its application in the design of children's wear [D]. Jiangnan University, 2017.

[7]. Li Hui. The application of court dress patterns in dress design in Qing Dynasty [D]. Northeast Normal University, 2016.

[8]. Li Jinxia. The aesthetic meaning of plant pattern in modern folk costume [D]. Jiangnan University, 2009.

[9]. Liang Huie, Xu Zheng Zheng. Geometric patterns and artistic charm of Chinese traditional costumes [J]. Hundred Schools in Arts , 2010,26 (S1): 185-187+184.

[10]. Sun Chengcheng, Liu Yuanfeng. A brief analysis of the formal features of court costumes in Qing Dynasty: taking animal pattern as an example, [J]. Art and Design Research, 2012 (S1): 4-7. 